\section{Tabagismo no domicílio e doença respiratória em crianças menores de cinco anos}

\author{
Household smoking and respiratory \\ disease in under-five children
}

\author{
1 Faculdade de Nutrição, \\ Universidade Federal de \\ Mato Grosso, Cuiabá, Brasil. \\ 2 Instituto de Medicina \\ Social, Universidade do \\ Estado do Rio de Janeiro, \\ Rio de Janeiro, Brasil. \\ 3 Escola Nacional de Saúde \\ Pública Sérgio Arouca, \\ Fundação Oswaldo Cruz, \\ Rio de Janeiro, Brasil. \\ Correspondência \\ R. M. V. Gonçalves-Silva \\ Faculdade de Nutrição, \\ Universidade Federal \\ de Mato Grosso. \\ Rua 27, casa 207, \\ Cuiabá, $M T$ \\ 78068-590, Brasil. \\ reveras@uol.com.br
}

\begin{abstract}
Environmental tobacco smoke is an important respiratory tract irritant in young children. To identify factors associated with respiratory disease and determine the main source of smoking exposure in the household, a cross-sectional study of 2,037 children who were immunized in primary health care clinics was conducted (in a sample of 10 out of 38 clinics with 200 children each). Parents answered $a$ questionnaire about children's birth, passive smoking, former and current respiratory morbidity, socio-demographic characteristics, and living conditions. Analysis was based on hierarchical logistic regression. Prevalence of respiratory symptoms was 59.9\% for children who live with smokers. Asthma and bronchitis showed the strongest association with smoking. In multivariate logistic regression, the following variables remained associated with asthma/bronchitis: socioeconomic status $(O R=$ 2.93; 95\%CI: 1.57-5.45), maternal schooling $(O R=$ 1.46; 95\%CI: 1.08-1.98)] and occupation (OR = 1.68; 95\%CI: 1.04-2.74), neighborhood (OR = 1.47; 95\%CI: 1.06-2.02), child's age (OR = 3.38; 95\%CI: 2.31-4.95) and sex (OR=1.46; 95\%CI: 1.09-1.94), breastfeeding $(O R=1.66$; 95\%CI: 1.15-2.40), and household smoking (OR = 1.58; 95\%CI: 1.18-2.11). Children with lower socioeconomic status and exposed to household smoking showed increased risk of respiratory disease.
\end{abstract}

Tobacco Smoke Pollution; Child; Respiratory Tract Diseases
Regina M. V. Gonçalves-Silva 1

Joaquim G. Valente 2,3

Márcia G. F. Lemos-Santos 1

Rosely Sichieri 2

\section{Introdução}

A infecção respiratória aguda é a principal causa de morte em crianças menores de cinco anos 1. Estimativas globais recentes indicam que morrem anualmente 10 milhões de crianças menores de cinco anos e que $99 \%$ dessas mortes ocorrem em países em desenvolvimento, $70 \%$ causadas por infecções, sendo as infecções do trato respiratório inferior a principal causa dos óbitos dessas crianças 2 .

No Brasil, a infecção respiratória aguda é também a maior responsável pela morbi-mortalidade de crianças com idade inferior a cinco anos 3,4 , constituindo, ainda, a principal causa de hospitalização dessa faixa etária 5 .

O aleitamento natural é considerado um fator protetor contra a infecção respiratória aguda 6 , e entre os fatores de risco incluem-se: a ventilação do domicílio, o estado nutricional da criança, o nível de escolaridade dos pais, a densidade domiciliar e o tabagismo dos moradores da casa, principalmente o da mãe 7,8.

A fumaça domiciliar do tabaco é o mais comum poluidor do ar doméstico, podendo variar sua concentração de acordo com o número de fumantes do domicílio e do número de cigarros fumados dentro de casa, estando associada a vários desfechos desfavoráveis à saúde infantil 9,10.

Apesar da evidência de que o fumo passivo aumenta a incidência das infecções respirató- 
rias e de já terem sido criadas leis (Lei n. 9.294 de 1996) proibindo o fumo em alguns locais públicos, são elevadas as taxas de exposição de crianças ao tabagismo passivo no Brasil 3,11,12,13. Com isso, um grande número de crianças continua sendo exposto ao tabagismo domiciliar.

Em Cuiabá, Mato Grosso, Brasil, já foram realizados alguns trabalhos, com população de clínicas, sobre os efeitos do fumo passivo na saúde de crianças menores de cinco anos 3,13. No entanto, não foram encontrados estudos de base populacional.

Diante do exposto, foi realizado este estudo com o objetivo de determinar quais os principais fatores associados aos sintomas e às enfermidades respiratórias de crianças de 0 a 5 anos e, ainda, qual a fonte de exposição ao tabagismo domiciliar que apresenta o maior risco para essas crianças.

\section{Métodos}

Foi realizado um estudo transversal, de base populacional, com crianças de ambos os sexos, com idade inferior a sessenta meses, que freqüentaram as salas de vacinação dos postos de saúde da Cidade de Cuiabá, entre agosto de 1999 e janeiro de 2000.

A cobertura vacinal da cidade é considerada alta (97\% para tríplice bacteriana em menores de um ano em 1999) e as crianças são encaminhadas pelo hospital em que nasceram ao posto de saúde mais próximo de sua residência para imunização. Dos 38 postos existentes, em quatro foram realizados estudos piloto com 400 crianças que indicaram prevalências de sintomas respiratórios como dispnéia, o menos freqüente, ao redor de $10 \%$. Para essa prevalência, com um poder de $80 \%$ e uma precisão de $1 \%$ seriam necessárias 1.934 crianças. Como a taxa de não resposta, no estudo piloto, foi muito baixa, a amostra foi constituída de $2 \mathrm{mil}$ crianças. Para tanto, foram sorteados dez postos com probabilidade proporcional ao volume de atendimento. Todos os responsáveis que estavam acompanhando os filhos foram convidados a participar do estudo. O recrutamento foi feito consecutivamente até atingir o total estimado de crianças em cada unidade sanitária. A taxa de recusa foi de $0,4 \%$.

Os pais ou responsáveis das crianças que concordaram em participar da pesquisa, responderam a um questionário contendo questões relacionadas ao nascimento das crianças, à exposição ao tabagismo passivo, à morbidade respiratória atual e pregressa, características sócio-demográficas e condições de moradia.
Os sinais e sintomas de infecção respiratória aguda foram referentes aos 15 dias anteriores à entrevista, para evitar possíveis vieses de memória. As enfermidades respiratórias, como bronquite ou asma e pneumonia, foram referidas pelo responsável. Para classificar os sintomas utilizou-se a Classificação Internacional de Doenças 14

A Cidade de Cuiabá é dividida em quatro regiões, conforme a área geográfica que ocupa, e em cada uma dessas regiões foi criado um distrito sanitário com o objetivo de gerir as unidades de saúde de sua região de abrangência. Dentre os quatro distritos existentes na cidade, o distrito oeste foi utilizado como referência pela menor prevalência de sintomas respiratórios entre as crianças.

Foram consideradas em aleitamento natural, as crianças cujas mães relataram estar amamentando no momento da entrevista.

O nível sócio-econômico foi avaliado pelo instrumento da Associação Brasileira de Institutos de Pesquisa de Mercado (ABIPEME) 15, em função de bens e escolaridade do chefe da família, com categorias que variam de A (nível mais elevado) até $\mathrm{E}$ (nível mais baixo). A ocupação dos pais foi classificada tendo como parâmetro a Classificação Brasileira de Ocupações 16 , sendo agrupadas aquelas cuja prevalência dos sintomáticos respiratórios era similar. Para os pais, a ocupação foi classificada em técnicas e artísticas; diretores e gerentes de empresas; administração; comércio; serventia; hospedagem; higiene e segurança; agropecuária; produção industrial e transportes. Para as mães, a ocupação foi classificada em técnicas e artísticas; administração; comércio; serventia; hospedagem; higiene e segurança; produção industrial.

A escolaridade dos pais foi classificada em quatro categorias: primeiro grau incompleto, primeiro grau completo, segundo grau completo e terceiro grau completo. Para analisar de forma combinada escolaridade e ocupação foi criada uma variável binária para escolaridade (mais baixa e mais elevada) com pontos de corte específicos para cada categoria ocupacional.

A renda mensal per capita da família foi obtida por meio da soma das rendas individuais dos moradores, dividida pelo total de moradores do domicílio, em salários mínimos. Para a análise da renda como variável categórica, foram utilizados a mediana e o terceiro quartil, como pontos de corte.

Foram considerados fumantes todos aqueles que, no momento da entrevista, referiram fumar pelo menos um cigarro por dia, pelo período mínimo de um ano, e tabagista gestacio- 
nal a mãe que informou ter fumado durante a gravidez.

Foi considerada exposta à fumaça domiciliar do tabaco ou fumante passivo, toda criança que morasse com pelo menos um fumante no domicílio (pais, parentes ou amigos).

\section{Análise dos dados}

Para a análise bivariada dos dados foi utilizado o teste do qui-quadrado para avaliar as diferenças estatísticas entre as proporções. Para verificar a linearidade das associações, utilizou-se o teste do qui-quadrado para tendência linear. A razão de prevalência foi usada como medida de associação entre a variável "presença de sintomas respiratórios" (variável dependente) e as demais variáveis estudadas. As variáveis do modelo teórico que se mostraram estatisticamente significativas na explicação das diferenças entre a presença ou não de sintomas respiratórios, na fase descritiva, foram selecionadas para a próxima fase da análise. Nesta etapa, por meio da regressão logística multivariada, as variáveis foram incluídas em blocos, utilizando a abordagem hierárquica 17 . Os fatores mais distais (sócio-econômicos e demográficos) foram os primeiros incluídos, seguidos das características familiares e do ambiente e, finalmente, aquelas mais próximas do evento. Foram testadas interações entre idade e as outras variáveis do modelo pela possibilidade de maior susceptibilidade entre os mais jovens, portanto, a idade seria modificadora de efeito das associações entre tabagismo com sintomas e doença.

Utilizou-se para as análises os programas Epi Info, versão 6.02 (Centers for Disease Control and Prevention, Atlanta, Estados Unidos), e SPSS versão 9.0 (SPSS Inc., Chicago, Estados Unidos).

A pesquisa foi aprovada pelo Comitê de Ética da Universidade Federal de Mato Grosso e pelos diretores dos distritos e gerentes dos postos de saúde, onde foram desenvolvidos o estudo. Antes do início da entrevista os pais ou responsáveis eram informados sobre o objetivo da pesquisa e da possibilidade de aceitar ou rejeitar a participação de seu filho na mesma. Após consentimento informado era dado início a coleta dos dados.

\section{Resultados}

Participaram do estudo 2.037 crianças com idade inferior a sessenta meses, sendo $51,4 \%$ do sexo masculino e $48,6 \%$ do feminino. Dentre essas, $49,4 \%$ eram menores de 12 meses, $32,6 \%$ tinham idade entre 12 e 36 meses e $18 \%$ eram maiores de 36 meses.

A prevalência de sintomáticos respiratórios nesse grupo foi de $59,9 \%$, sendo maior para os que conviviam com fumantes. O "tabagismo dos moradores" foi a variável que se mostrou mais associada com a presença de sintomáticos respiratórios no domicílio, sendo por isso considerado marcador do tabagismo no domicílio.

Os principais sinais, sintomas e infecção respiratória aguda estão descritos na Tabela 1. Dentre as patologias pulmonares referidas, a

Tabela 1

Prevalência, razão de prevalência (RP) e intervalo de confiança (IC95\%) de sinais, sintomas e infecção respiratória aguda, nos 15 dias anteriores à entrevista, em crianças menores de cinco anos, segundo o tabagismo no domicílio. Cuiabá, Mato Grosso, Brasil, 1999.

\begin{tabular}{|c|c|c|}
\hline Exposição ao tabagismo & Prevalência & RP (IC95\%) \\
\hline \multicolumn{3}{|l|}{ Sintomáticos respiratórios } \\
\hline Não & $56,9(716 / 1.269)$ & 1,00 \\
\hline Sim & $65,6(504 / 768)$ & $1,16(1,08-1,25)$ \\
\hline \multicolumn{3}{|l|}{ Tosse } \\
\hline Não & $37,1(472 / 1.269)$ & 1,00 \\
\hline Sim & $46,2(354 / 768)$ & $1,24(1,12-1,38)$ \\
\hline \multicolumn{3}{|l|}{ Dispnéia } \\
\hline Não & $14,5(184 / 1.267)$ & 1,00 \\
\hline Sim & $18,9(137 / 766)$ & $1,23(1,01-1,51)$ \\
\hline \multicolumn{3}{|l|}{ Coriza } \\
\hline Não & $35,9(456 / 1.269)$ & 1,00 \\
\hline Sim & $43,5(334 / 768)$ & $1,21(1,09-1,35)$ \\
\hline \multicolumn{3}{|l|}{ Chiado } \\
\hline Não & $24,1(305 / 1.269)$ & 1,00 \\
\hline Sim & $28,1(215 / 768)$ & $1,21(0,96-1,52)$ \\
\hline \multicolumn{3}{|l|}{ Dor de ouvido } \\
\hline Não & $7,8(99 / 1.269)$ & 1,00 \\
\hline $\operatorname{Sim}$ & $8,3(64 / 768)$ & $1,07(0,79-1,44)$ \\
\hline \multicolumn{3}{|l|}{ Asma/Bronquite referida } \\
\hline Não & $9,1(116 / 1.269)$ & 1,00 \\
\hline Sim & $15,9(122 / 768)$ & $1,75(1,37-2,20)$ \\
\hline \multicolumn{3}{|l|}{ Pneumonia referida } \\
\hline Não & $10,9(138 / 1.269)$ & 1,00 \\
\hline Sim & $11,7(90 / 768)$ & $1,08(0,84-1,38)$ \\
\hline \multicolumn{3}{|c|}{ Internação na vida por pneumopatia } \\
\hline Não & $10,6(134 / 1.269)$ & 1,00 \\
\hline Sim & $13,2(101 / 768)$ & $1,25(0,98-1,59)$ \\
\hline
\end{tabular}


asma/bronquite foi a que apresentou maior associação com o tabagismo, representando uma chance quase duas vezes maior para os expostos em relação aos não-expostos.

Do total de crianças que nasceram prematuramente, $8,5 \%$ pertenciam a famílias onde existiam fumantes e 5,3\% a não-fumantes (risco relativo $-\mathrm{RR}=1,65$; intervalo de confiança de 95\% - IC95\%: 1,19-2,29).

Os filhos de pais mais jovens, menos escolarizados, pertencentes a famílias de menor nível socioeconômico e residentes em moradias com piores condições ambientais, mostraram maior prevalência de problemas respiratórios. Além disso, filhos de empregadas domésticas e crianças com idade igual ou maior do que um ano também apresentaram maior probabilidade de apresentar sintomas respiratórios (Tabela 2).

Dentre todas as fontes de exposição ao tabagismo domiciliar, a que apresentou maior associação com a presença de sintomáticos respiratórios na família foi o tabagismo de qualquer morador (Tabela 3), sendo, portanto, utilizado como marcador para exposição ao tabagismo domiciliar pelas crianças.

A Tabela 4 apresenta os resultados para os sintomas respiratórios da regressão logística hierarquizada. Crianças com idade igual ou maior do que um ano apresentaram uma probabilidade $30 \%$ maior de ter sintomas respiratórios do que as menores de um ano (odds ratio - OR = 1,29; IC95\%: 1,08-1,56). Com a inclusão no modelo, no nível 4 , da variável tabagismo dos moradores e do termo de interação, tabagismo dos moradores e idade da criança, observou-se um efeito para tabagismo dos moradores em menores de um ano de 1,67 (IC95\%: 1,26-2,23) e para tabagismo dos moradores em crianças com um ano e mais de 1,04 (IC95\%: $0,79-1,36$ )

Dentre as variáveis sócio-econômicas que permaneceram associadas com o desfecho, o salário per capita teve um efeito protetor para os sintomas respiratórios. Em relação às variáveis ambientais apenas o distrito permaneceu no modelo, mostrando que a criança que nasceu no distrito norte tem quase o triplo da chance de ter doença respiratória do que aquela nascida no distrito oeste.

Para a asma/bronquite como variável resposta (Tabela 5), os resultados encontrados para as variáveis sócio-econômicas foram similares às do modelo anterior, com a diferença de que neste, o nível sócio-econômico esteve fortemente relacionado com a presença de asma/bronquite, mostrando que crianças pertencentes ao nível sócio-econômico mais baixo (E) apresentavam uma chance quase três vezes maior de ter asma/bronquite do que aquelas pertencentes aos níveis mais elevados (A ou B).

Em relação às variáveis biológicas, verificase uma maior associação para o sexo masculino, para as crianças que não receberam aleitamento natural e para as maiores de um ano. É importante ressaltar que quando a variável idade entrou no modelo como contínua, a chance de a criança apresentar asma/bronquite aumentou em 3\% ao mês (OR = 1,03; IC95\%: 1,02$1,04)$.

\section{Discussão}

Os resultados deste estudo mostram que o tabagismo dos moradores do domicílio foi a variável que apresentou a maior associação com a doença respiratória. Apesar do forte impacto do tabagismo da mãe e do pai sobre os sintomas respiratórios, nota-se que quando se associa o tabagismo dos pais e dos moradores, são mais graves os resultados produzidos sobre a saúde respiratória das crianças, sugerindo um aumento na dose de exposição. Resultados semelhantes foram encontrados por Ownby et al. 18, os quais estudando a exposição passiva de crianças de até dois anos de idade, verificaram que o fumo de outros moradores, além dos pais, contribuiu de forma significante para a doença respiratória.

Neste trabalho, a preferência pelo estudo de lactentes e pré-escolares se deve ao fato de que essas crianças permanecem por um período mais longo de tempo dentro de casa, em íntimo contato com os adultos, sendo expostas mais intensamente do que crianças mais velhas, que já freqüentam escolas.

Embora existam métodos mais acurados para aferir a exposição passiva ao tabaco, utilizou-se o questionário, por não ser invasivo, de baixo custo e de fácil execução 19,20. Trabalhos realizados comparando valores da excreção urinária de cotinina com dados de questionário sobre o hábito de fumar dos pais mostraram-se fortemente correlacionados 21,22 .

Pode ter havido, neste estudo, erros de classificação quanto à exposição das crianças ao tabagismo, já que uma informação obtida transversalmente foi utilizada para avaliar desfechos ao longo da vida da criança. Uma outra possível limitação são os erros quanto aos sintomas e doenças referidas pelo responsável.

Identificou-se que os fatores associados para sintomas respiratórios em geral e asma/bronquite foram semelhantes, muito embora o modelo que utilizou a asma/bronquite, como va- 
riável dependente, tenha apresentado uma associação mais forte com o nível sócio-econômico mais baixo. A criança de família da categoria mais baixa (E) apresentou chance quase três vezes maior de vir a apresentar asma/ bronquite do que a criança pertencente aos níveis mais elevados (A ou B).

Em relação à idade da criança, observa-se que, a probabilidade de apresentar sintomas respiratórios era maior para aquelas com um ano de idade ou mais. Isto pode ter ocorrido porque nestes domicílios era mais elevado o número de moradores fumantes do que naqueles onde viviam crianças com idade inferior a um ano (dados não apresentados). Outra explicação para este achado é a maior freqüência com que as crianças são levadas às consultas médicas no primeiro ano de vida. A maior proximidade da mãe com o serviço de saúde pode favorecer a mudança de comportamento dos pais e até dos moradores do domicílio em relação ao hábito de fumar.

Por outro lado, a interação encontrada entre as variáveis tabagismo dos moradores e a idade da criança, mostrou que quando são menores de um ano e ainda expostas ao tabagismo dos moradores, a chance de apresentarem sintomas respiratórios é 1,7 vez maior do que para aquelas com idade igual ou maior do que um ano. Essa tendência é atribuída, geralmente, à aquisição de imunidade específica crescente para um maior grupo de vírus que ocorre naturalmente com a idade 23 .

O estudo da associação entre o sexo das crianças e a morbidade respiratória, mostrou que, os sintomas respiratórios, por serem muitas vezes inespecíficos, ocorrem nas crianças independentemente do sexo, o que já não se observa para asma/bronquite. Neste caso, os meninos apresentaram maior probabilidade para a doença do que as meninas. Embora vários estudos tenham apresentado resultados semelhantes a este $1,24,25,26$, a razão para a maior susceptibilidade dos meninos não é conhecida.

Quanto ao aleitamento, a criança que não estava sendo amamentada apresentava uma chance 1,7 vez maior de apresentar asma/bronquite do que as que estavam sendo amamentadas. Outros estudos também mostraram que o aleitamento natural é um fator de proteção para as infecções respiratórias 6,27,28. Este efeito pode ser decorrente de uma combinação de mecanismos comportamentais, que podem produzir mudanças relacionadas à exposição da criança ao tabagismo domiciliar, e biológicos, por meio da aquisição de imunidade específica crescente para um maior grupo de vírus,
Tabela 2

Prevalência, razão de prevalência (RP) e intervalo de confiança (IC95\%) de sintomáticos respiratórios, nos 15 dias anteriores à entrevista, em crianças menores de cinco anos, segundo variáveis sócio-demográficas e ambientais. Cuiabá, Mato Grosso, Brasil, 1999.

\begin{tabular}{|c|c|c|}
\hline Variáveis & Prevalência & RP (IC95\%) \\
\hline \multicolumn{3}{|l|}{ Nível 1} \\
\hline $\begin{array}{l}\text { Nível sócio-econômico } \\
\qquad \begin{array}{l}A+B+C \text { (+ elevado) } \\
D+E(+ \text { baixo })\end{array}\end{array}$ & $\begin{array}{c}57,0(700 / 1.227) \\
64,2(520 / 810)\end{array}$ & $\begin{array}{c}1,00 \\
1,13(1,05-1,21)\end{array}$ \\
\hline $\begin{array}{l}\text { Renda per capita (salários } \\
\begin{array}{l}2,5 \text { e }+ \\
1-2,4 \\
<1\end{array}\end{array}$ & $\begin{array}{c}49,4(166 / 336) \\
58,1(352 / 606) \\
64,1(702 / 1.095)\end{array}$ & $\begin{array}{c}1,00 \\
1,18(1,03-1,34) \\
1,30(1,15-1,46)\end{array}$ \\
\hline $\begin{array}{l}\text { Escolaridade da mãe } \\
\text { + elevada } \\
\text { + baixa }\end{array}$ & $\begin{array}{c}53,7(516 / 961) \\
65,3(674 / 1.032)\end{array}$ & $\begin{array}{c}1,00 \\
1,22(1,13-1,31)\end{array}$ \\
\hline $\begin{array}{l}\text { Idade da mãe (anos) } \\
\qquad 25 \text { e }+ \\
<25\end{array}$ & $\begin{array}{c}56,2(560 / 997) \\
63,4(647 / 1.020)\end{array}$ & $\begin{array}{c}1,00 \\
1,13(1,05-1,21)\end{array}$ \\
\hline $\begin{array}{l}\text { Ocupação da mãe } \\
\text { Outras } \\
\text { Empregada doméstica }\end{array}$ & $\begin{array}{c}59,0(1.119 / 1.897) \\
73,3(88 / 120)\end{array}$ & $\begin{array}{c}1,00 \\
1,24(1,11-1,39)\end{array}$ \\
\hline $\begin{array}{l}\text { Escolaridade do pai } \\
\text { + elevada } \\
\text { + baixa }\end{array}$ & $\begin{array}{l}54,9(412 / 751) \\
61,4(515 / 839)\end{array}$ & $\begin{array}{c}1,00 \\
1,12(1,03-1,22)\end{array}$ \\
\hline $\begin{array}{l}\text { Idade do pai (anos) } \\
29 \mathrm{e}+ \\
<29\end{array}$ & $\begin{array}{l}57,5(495 / 861) \\
60,0(478 / 797)\end{array}$ & $\begin{array}{c}1,00 \\
1,04(0,96-1,13)\end{array}$ \\
\hline $\begin{array}{l}\text { Ocupação do pai } \\
\text { Outras } \\
\text { Indústria }\end{array}$ & $\begin{array}{c}56,3(615 / 1.092) \\
63,3(358 / 566)\end{array}$ & $\begin{array}{c}1,00 \\
1,12(1,03-1,22)\end{array}$ \\
\hline Nível 2 & & \\
\hline $\begin{array}{l}\text { Material da casa } \\
\text { Tijolo } \\
\text { Outro }\end{array}$ & $\begin{array}{c}59,4(1.142 / 1.924) \\
69,0(78 / 113)\end{array}$ & $\begin{array}{c}1,00 \\
1,16(1,02-1,32)\end{array}$ \\
\hline $\begin{array}{l}\text { Densidade domiciliar } \\
\quad \leq 2 \\
>2\end{array}$ & $\begin{array}{c}59,4(1.134 / 1.913) \\
67,7(84 / 124)\end{array}$ & $\begin{array}{c}1,00 \\
1,14(1,00-1,30)\end{array}$ \\
\hline $\begin{array}{c}\text { Distrito } \\
\text { Oeste } \\
\text { Leste } \\
\text { Sul } \\
\text { Norte }\end{array}$ & $\begin{array}{l}46,6(282 / 605) \\
57,9(356 / 615) \\
68,1(280 / 411) \\
74,4(302 / 406)\end{array}$ & $\begin{array}{c}1,00 \\
1,24(1,11-1,38) \\
1,46(1,31-1,63) \\
1,60(1,44-1,77)\end{array}$ \\
\hline Nível 3 & & \\
\hline $\begin{array}{l}\text { Sexo da criança } \\
\text { Masculino } \\
\text { Feminino }\end{array}$ & $\begin{array}{c}59,8(626 / 1.047) \\
60,0(594 / 990)\end{array}$ & $\begin{array}{c}1,00 \\
1,00(0,93-1,08)\end{array}$ \\
\hline $\begin{array}{l}\text { Idade da criança (meses) } \\
\quad<12 \\
12 \mathrm{e}+\end{array}$ & $\begin{array}{l}56,1(564 / 1.006) \\
63,6(656 / 1.031)\end{array}$ & $\begin{array}{c}1,00 \\
1,13(1,06-1,22)\end{array}$ \\
\hline $\begin{array}{l}\text { Aleitamento materno } \\
\text { Sim } \\
\text { Não }\end{array}$ & $\begin{array}{c}57,1(517 / 905) \\
62,1(703 / 1.132)\end{array}$ & $\begin{array}{c}1,00 \\
1,09(1,01-1,17)\end{array}$ \\
\hline
\end{tabular}

* $p<0,0001$ (para tendência linear). 
Tabela 3

Prevalência, razão de prevalência (RP) e intervalo de confiança (IC95\%) dos sintomáticos respiratórios, segundo a fonte de exposição ao tabagismo domiciliar. Cuiabá, Mato Grosso, Brasil, 1999

\begin{tabular}{lcc}
\hline Fonte de exposição & $\begin{array}{c}\text { Sintomáticos } \\
\text { respiratórios } \\
\text { Prevalência (\%) }\end{array}$ & RP (IC 95\%) \\
\hline Tabagismo na gestação & $59,3(1.085 / 1.829)$ & 1,00 \\
$\quad$ Não & $64,9(135 / 208)$ & $1,09(0,98-1,22)$ \\
Sim & $59,1(1.056 / 1.788)$ & 1,00 \\
Tabagismo da mãe & $66,0(151 / 229)$ & $1,12(1,01-1,23)$ \\
Não & $57,5(721 / 1.255)$ & 1,00 \\
Sim & $63,3(255 / 403)$ & $1,10(1,01-1,20)$ \\
Tabagismo do pai & & 1,00 \\
Não & $56,4(716 / 1.269)$ & $1,16(1,08-1,25)$ \\
Sim & $65,6(504 / 768)$ & \\
Tabagismo dos moradores & & \\
Não &
\end{tabular}

Tabela 4

Odds ratio (OR) ajustada e intervalo de confiança de 95\% (IC95\%), em modelo hierárquico, para os fatores associados aos sintomas respiratórios de crianças menores de cinco anos. Cuiabá, Mato Grosso, Brasil, 1999.

\begin{tabular}{|c|c|}
\hline Variáveis & OR ajustada (IC95\%) \\
\hline \multicolumn{2}{|l|}{ Nível 1} \\
\hline Salário per capita & $0,94(0,90-0,98)$ \\
\hline \multicolumn{2}{|l|}{ Escolaridade da mãe } \\
\hline Mais baixa/Mais alta & $1,43(1,18-1,73)$ \\
\hline \multicolumn{2}{|l|}{ Ocupação da mãe } \\
\hline Empregada doméstica/Demais & $1,63(1,07-2,49)$ \\
\hline \multicolumn{2}{|l|}{ Nível 2} \\
\hline \multicolumn{2}{|l|}{ Distrito sanitário } \\
\hline Norte/Oeste & $2,87(2,15-3,83)$ \\
\hline Sul/Oeste & $2,11(1,60-2,79)$ \\
\hline Leste/Oeste & $1,40(1,09-1,78)$ \\
\hline \multicolumn{2}{|l|}{ Nível 3} \\
\hline \multicolumn{2}{|l|}{ Idade da criança (anos) } \\
\hline $1 e+/<1$ & $p<0,01$ \\
\hline \multicolumn{2}{|l|}{ Nível 4} \\
\hline \multicolumn{2}{|l|}{ Tabagismo dos moradores } \\
\hline Sim/Não & $p<0,01$ \\
\hline Tabagismo dos moradores $\mathrm{x}$ idade da criança & $p<0,01$ \\
\hline$<12$ & $1,69(1,26-2,27)$ \\
\hline $12 \mathrm{e}+$ & $1,04(0,79-1,37)$ \\
\hline
\end{tabular}

que se observa quando a criança está sendo aleitada 29.

Em relação às variáveis sócio-econômicodemográficas, os resultados apontam, claramente, para uma relação entre o nível sócioeconômico mais baixo e o aumento da predisposição para doenças respiratórias. Assim, fatores sócio-ambientais desfavoráveis como a elevada densidade domiciliar, habitações insalubres que apresentam maior poluição ambiental, inclusive com maior prevalência de tabagismo entre os moradores, e menor acesso à assistência médica são fatores que podem contribuir individualmente ou talvez interagir para aumentar a susceptibilidade das infecções respiratórias desses grupos.

Em relação à escolaridade dos pais, apenas a escolaridade da mãe manteve-se associada à doença respiratória no modelo final. A educação da mãe é um forte preditor da saúde da criança, o que vem reforçar a idéia de que talvez esse fator seja o mais importante determinante sócio-econômico das condições de saúde e doença da criança 30,31. Mães mais escolarizadas podem mudar seu hábito de fumar ou adotar padrões comportamentais em relação ao fumo diferentes daquelas de menor escolaridade, como, por exemplo, não fumar dentro de casa, não fumar na presença dos filhos, impedir que outros moradores fumem na presença das crianças, por saber que esta atitude pode prejudicar a saúde infantil.

A renda familiar per capita também foi uma variável importante na determinação dos sintomas respiratórios das crianças. A renda familiar influencia a qualidade de vida. Assim, famílias com renda per capita mais elevada têm a possibilidade de cuidar mais ativamente da saúde dos filhos, o que pode resultar em menor ocorrência de doenças 32 .

Da mesma forma, o escore utilizado para classificar as famílias em cinco categorias distintas de nível sócio-econômico mostrou maior prevalência de sintomas respiratórios nos níveis mais baixos. Após ajustar-se para fatores de confusão, verificou-se que o nível mais baixo triplicou a chance de a criança apresentar asma/bronquite, o que mostra, mais uma vez, a importância do nível sócio-econômico como determinante da saúde infantil.

Em relação às variáveis ambientais, observa-se que a região onde a criança vive, representada neste estudo pelo distrito, apresentou associação significante com a doença respiratória. A ocorrência de doenças respiratórias costuma ser maior em ambientes insalubres, em conseqüência de uma situação sócio-econômica desfavorável 33 . 
Tendo em vista a expressiva prevalência de sintomas respiratórios encontrada nos menores de cinco anos deste estudo, e, considerando que em alguns períodos do ano, a Cidade de Cuiabá torna-se muito poluída devido ao período das queimadas, é importante que os órgãos responsáveis pela saúde pública despertem para a necessidade da melhoria da qualidade de vida desse grupo da população.

Sabendo-se que o tabagismo é um elemento nocivo às vias aéreas das crianças, esforços devem ser envidados no sentido de que não apenas os pais, mas também os demais moradores dos domicílios se abstenham de fumar, pelo menos na presença das crianças, de forma a reduzir os efeitos deletérios à saúde infantil. Programas de educação para a saúde devem ser fomentados visando a esse objetivo.

\section{Resumo}

A fumaça do tabaco no ambiente doméstico éo principal irritante do trato respiratório de crianças. Avaliaram-se os fatores associados aos sintomas e às enfermidades respiratórias, assim como a principal fonte de exposição ao tabagismo no domicílio de 2.037 crianças menores de cinco anos atendidas para vacinação em 10 dos 38 postos de saúde de Cuiabá, Mato Grosso, Brasil (aproximadamente duzentas crianças por posto). Exposição ao tabagismo, a morbidade respiratória e as características sócio-demográficas foram obtidas por entrevista com o responsável pela criança. A prevalência de sintomáticos respiratórios foi de 59,9\%, sendo maior para os que convivem com fumantes. A asma/bronquite foi a patologia que esteve mais fortemente associada com o tabagismo, sendo as variáveis que permaneceram associadas em modelo logístico hierarquizado: nível socioeconômico $(O R=$ 2,93; IC95\%: 1,57-5,45), escolaridade (OR = 1,46; IC95\%: 1,08-1,98) e ocupação da mãe (OR = 1,68; IC95\%: 1,04$2,74)$, distrito sanitário $(R P=1,47 ;$ IC95\%: 1,06-2,02), faixa etária $(\mathrm{OR}=3,38$; IC95\%: 2,31-4,95) e sexo da criança $(O R=1,46$; IC95\%: 1,09-1,94), aleitamento natural (OR = 1,66; IC95\%: 1,15-2,40) e tabagismo dos moradores $(O R=1,58$; IC95\%: 1,18-2,11). Crianças pertencentes aos níveis socioeconômicos mais baixos e expostas ao tabagismo dos moradores do domicílio apresentam maior associação com a doença respiratória.

Poluição por Fumaça de Tabaco; Criança; Doenças Respiratórias

\section{Colaboradores}

Todos os autores participaram das fases de elaboração, análise e interpretação dos dados.
Tabela 5

Odds ratio (OR) ajustada e intervalo de confiança de 95\% (IC95\%), em modelo hierárquico, para os fatores associados à asma/bronquite de crianças menores de cinco anos, Cuiabá, Mato Grosso, Brasil, 1999.

\begin{tabular}{lc}
\hline Variáveis & OR ajustada (IC95\%) \\
\hline Nível 1 & \\
Nível sócio-econômico* & \\
C + D/A + B & $1,32(0,83-2,10)$ \\
E/A + B & $2,93(1,57-5,45)$ \\
Escolaridade da mãe & \\
$\quad$ Mais baixa/Mais alta & $1,46(1,08-1,98)$ \\
Ocupação da mãe & \\
Empregada doméstica/Demais & $1,68(1,04-2,74)$
\end{tabular}

Nível 2

Distrito sanitário

Norte/Sul + Leste + Oeste

$1,47(1,06-2,02)$

Nível 3

Sexo

Masculino/Feminino

$1,46(1,09-1,94)$

Aleitamento natural

Não/Sim

$1,66(1,15-2,40)$

Idade da criança (anos)

1 e $+/<1$

$3,38(2,31-4,95)$

Nível 4

Tabagismo dos moradores

Sim/Não

$1,58(1,18-2,11)$

* Classificação segundo a Associação Brasileira de Institutos de Pesquisa de Mercado 15.

\section{Referências}

1. Broor S, Pandey RM, Ghosh M, Maitreyi RS, Lodha R, Singhal T, et al. Risk factors for severe acute lower respiratory tract infection in under-five children. Indian Pediatr 2001; 38:1361-9.

2. Adegbola RA, Obaro SK. Diagnosis of childhood pneumonia in the tropics. Ann Trop Med Parasitol 2000; 94:197-207.

3. Duarte DMG, Botelho C. Perfil clínico de crianças menores de cinco anos com infecção respiratória aguda. J Pediatr (Rio J) 2000; 76:207-12.

4. Straliotto SM, Siqueira MM, Muller RL, Fischer GB, Cunha MLT, Nestor SM. Viral etiology of acute respiratory infections among children in Porto Alegre, RS, Brazil. Rev Soc Bras Med Trop 2002; 35:283-91.

5. Caetano JRM, Bordin IAS, Puccini RF, Peres CA. Fatores associados à internação hospitalar de crianças menores de cinco anos, São Paulo, SP. Rev Saúde Pública 2002; 36:285-91. 
6. Oddy WH, Sly PD, de Klerk NH, Landau LI, Kendall GE, Holt PG, et al. Breast feeding and respiratory morbidity in infancy: a birth cohort study. Arch Dis Child 2003; 88:224-8.

7. Stoddard JJ, Miller T. Impact of parental smoking on the prevalence of wheezing respiratory illness in children. Am J Epidemiol 1995; 141:96-102.

8. Cunha AL. Relationship between acute respiratory infection and malnutrition in children under 5 years of age. Acta Paediatr 2000; 89:608-9.

9. Difranza JR, Lew RA. Morbidity and mortality in children associated with the use of tobacco products by other people. Pediatrics 1996; 97:560-8.

10. Adair-Bischoff CE, Sauve RS. Environmental tobacco smoke and middle ear disease in preschoolage children. Arch Pediatr Adolesc Med 1998; 152:127-33.

11. Botelho C, Barbosa LSG, Silva MD, Barros MD. Sintomas respiratórios e tabagismo passivo em crianças. J Pneumol 1987; 13:136-43.

12. Pereira EDB, Torres L, Macedo J, Medeiros MMC. Efeitos do fumo ambiental no trato respiratório inferior de crianças com até 5 anos de idade. Rev Saúde Pública 2000; 34:39-43.

13. Botelho C, Correia AL, Silva AMC, Macedo AG, Silva COS. Fatores ambientais e hospitalizações em crianças menores de cinco anos com infecção respiratória aguda. Cad Saúde Pública 2003; 19: 1771-80.

14. World Health Organization. ICD-10: international statistical classification of diseases and related health problems. 10 th Rev. Geneva: World Health Organization; 1992.

15. Associação Brasileira dos Institutos de Pesquisa de Mercado. Proposição para um novo critério de classificação sócio-econômica, 1978. São Paulo: Associação Brasileira dos Institutos de Pesquisa de Mercado; 1978.

16. Secretaria de Políticas de Emprego e Salário, Ministério do Trabalho. Tábua de conversão CBO 94. Brasília: Secretaria de Políticas de Emprego e Salário, Ministério do Trabalho; 1996.

17. Victora CG, Huttly SR, Fuchs SC, Olinto MTA. The role of conceptual frameworks in epidemiological analysis: a hierarchical approach. Int J Epidemiol 1997; 26:224-7.

18. Ownby DR, Johnson CC, Peterson EL. Passive cigarette smoke exposure of infants: importance of nonparental sources. Arch Pediatr Adolesc Med 2000; 154:1237-41.

19. Gilliland FD, Li YF, Peters JM. Effects of maternal smoking during pregnancy and environmental tobacco smoke on asthma and wheezing in children. J Respir Crit Care Méd 2001; 163:429-36.

20. Carvalho LMT, Pereira EDB. Morbidade respiratória em crianças fumantes passivas. J Pneumol 2002; 28:8-14.
21. Slattery ML, Hunt SC, French TK, Ford MH, Williams RR. Validity of cigarette smoking habits in three epidemiologic studies in Utah. Prev Med 1989; 18:11-9.

22. Henschen M, Frischer T, Pracht T, Spiekerkötter E, Karmaus W, Meinert R, et al. The internal dose of passive smoking at home depends on the size of the dwelling. Environ Res 1997; 72:65-71.

23. Graham NMH. The epidemiology of acute respiratory infections in children and adults: a global perspective. Epidemiol Rev 1990; 12:149-78.

24. Schwartz J, Gold D, Dockery DW, Weiss ST, Speizer FE. Predictors of asthma and persistent wheeze in a national sample of children in the United States. Association with social class, perinatal events, and race. Am Rev Respir Dis 1990; 142:55562.

25. Marbury MC, Maldonado G, Walter L. The indoor air and children's health study: methods and incidence rates. Epidemiology 1996; 7:166-74.

26. Chatkin M, Menezes AM, Albernaz E, Victora CG, Barros FC. Fatores de risco para consultas em pronto-socorro por crianças asmáticas no Sul do Brasil. Rev Saúde Pública 2000; 34:491-8.

27. Blaymore Bier JA, Oliver T, Ferguson A, Vohr BR. Human milk reduces outpatient upper respiratory symptoms in premature infants during their first year of life. J Perinatol 2002; 22:354-9.

28. Chulada PC, Arbes Jr. SJ, Dunson D, Zeldin DC. Breast-feeding and the prevalence of asthma and wheeze in children: analyses from the Third National Health and Nutrition Examination Survey, 1988-1994. J Allergy Clin Immunol 2003; 111:32836.

29. Woodward A, Douglas RM, Graham NM, Miles H. Acute respiratory illness in Adelaide children: breast feeding modifies the effect of passive smoking. J Epidemiol Community Health 1990; 44:22430.

30. Kriz P, Bobak M, Kriz B. Parental smoking, socioeconomic factors, and risk of invasive meningococcal disease in children: a population based casecontrol study. Arch Dis Child 2000; 83:117-21.

31. Prietsch SOM, Fischer GB, Cesar JA, Fabris AR, Mehanna H, Ferreira THP, et al. Doença aguda das vias aéreas inferiores em menores de cinco anos: influência do ambiente doméstico e do tabagismo materno. J Pediatr (Rio J) 2002; 78:415-22.

32. Cesar JA, Horta BL, Gomes G, Shehadeh I, Chitolina J, Rangel L, et al. Utilização de serviços de saúde por menores de cinco anos no extremo Sul do Brasil. Cad Saúde Pública 2002; 18:299-305.

33. Peters J, Hedley AJ, Wong CM, Lam TH, Ong SG, Liu J, et al. Effects of an ambient air pollution intervention and environmental tobacco smoke on children's respiratory health in Hong Kong. Int J Epidemiol 1996; 25:821-8.

Recebido em 05/Ago/2004

Versão final reapresentada em 22/Dez/2004 Aprovado em 13/Jun/2005 